\title{
The role of pharmacogenetics of cytochrome P450s in phenytoin-induced DRESS syndrome
}

\author{
$\ddot{U M I T}$ YAŞAR \\ Department of Medical Pharmacology, Faculty of Medicine, Hacettepe University, Ankara, Turkey
}

\begin{abstract}
Anil et al. (2017) report a patient who presented with drug rash with eosinophilia and systemic symptom (DRESS) syndrome. It may be induced by various drugs. In the mentioned report DRESS syndrome has been attributed to phenytoin use.

CYP2C9 is a genetically polymorphic enzyme, and decreased metabolism of many drugs has been reported in the subjects carrying variants of rs 1799853 and rs1057910, which were designated as CYP2C9*2 and *3. rs3758581, the variant investigated by Anil et al., is a genetic variant of CYP2C19 (not that of CYP2C9, as stated and discussed in the report). CYP2C19 is also a highly genetically polymorphic enzyme, and rs3758581 may be present in 40 different haplotypes of CYP2C19 including $* 2$ and *17 (for detailed information: www.pharmvar.org/gene/CYP2C19). Detection of rs3758581 is not sufficient because the patient presented by Anil H et al. may have CYP2C19*2, CYP2C19*17, or any other variant due to the co-appearance of other possible genetic variations. It is of importance to perform the correct genetic analysis because CYP2C19*2 is associated with low enzyme activity but $C Y P 2 C 19 * 17$ is associated with increased enzyme activity.

CYP2C9 is a major enzyme responsible for the metabolism of phenytoin. CYP2C9*2 (rs 1799853) and $* 3$ (rs1057910) should be considered in a Caucasian subject. CYP2C19 (especially *2 and *17) and ABCB1 polymorphisms may also be considered for the evaluation of the patient. Additionally, serum phenytoin levels would be helpful to understand the contribution of genetic polymorphisms on the pharmacokinetics of phenytoin in the patients presenting side effects like DRESS syndrome.
\end{abstract}

Key words: phenytoin, genetic polymorphism, DRESS syndrome, cytochrome P450.

(Centr Eur Immunol 2018; 43 (2): 220-221)

Anil et al. (2017) report a patient who presented drug rash with eosinophilia and systemic symptom (DRESS) syndrome [1]. Various drugs may induce DRESS syndrome as stated by Anil et al. [1,2]. In the mentioned report DRESS syndrome has been attributed to phenytoin use [1].

As mentioned in the report, phenytoin is mainly metabolised by cytochrome P450 (CYP) 2C9. This CYP is a genetically polymorphic enzyme, and decreased metabolism of many drugs, for example phenytoin, celecoxib, and losartan, have been reported in subjects carrying variants of rs1799853 and rs1057910, which were designated as CYP2C9*2 and $* 3$ at the official website of CYP nomenclature, www.pharmvar.org, respectively [3-5]. *2 and *3 are the most common variant alleles of CYP2C9 in Caucasians including the Turkish population [4].

rs3758581, the variant investigated by Anil et al., is a genetic variant of CYP2C19 (not that of CYP2C9, as stated and discussed in the report [1]). CYP2C19 is also a highly genetically polymorphic enzyme, and rs3758581 may be present in 40 different haplotypes of CYP2C19 including *2 and *17 (for detailed information: www.pharmvar.org/ gene/CYP2C19). Among these haplotypes CYP2C19*2 and CYP2C19*17 are the most common variants in Caucasians, including the Turkish population [6]. Detection of rs3758581 is insufficient because the patient presented by Anil $\mathrm{H}$ et al. may have CYP2C19*2, CYP2C19*17, or any other variant due to the co-appearance of other possible genetic variations. It is of importance to perform the correct genetic analysis because CYP2C19*2 is associated with low enzyme activity, but CYP2C19*17 is associated with increased enzyme activity [6].

Although the rs3758581 analysed in the patient was not attributed to the correct gene, it is not totally wrong to genotype CYP2C19 for a suspected phenytoin toxicity because genetic polymorphisms of the CYP2C19 are partly related with phenytoin in some studies [5].

Correspondence: Ümit Yaşar, MD PhD, Department of Medical Pharmacology, Faculty of Medicine, Hacettepe University, 06100 Ankara,

Turkey, e-mail: uyasar@hacettepe.edu.tr

Submitted: 27.03.2018; Accepted: 10.04.2018 
It is also important to clarify if the patient is a heterozygous or homozygous carrier of the variant. This has not been mentioned in the report. Subjects who are homozygous carriers of CYP2C $9 * 3$ have almost no enzyme activity whereas heterozygous carriers have $50 \%$ of normal activity [6].

Another important issue is detection of serum phenytoin concentrations, which could have been valuable in this patient. In the literature, there are cases with lack of enzyme activity, in which serum phenytoin concentrations decreased to therapeutic ranges from toxic levels more than a week after stopping the treatment [7].

Another point is that phenytoin is a substrate of p-glycoprotein, which is also genetically polymorphic. P-glycoprotein is coded by $A B C B 1$ gene. Phenytoin disposition is also affected by polymorphisms of $A B C B 1$ [8]. This could have been taken into account for phenytoin toxicity.

In conclusion, CYP2C9 is a major enzyme responsible from the metabolism of phenytoin. CYP2C $9 * 2$ (rs1799853) and $* 3$ (rs1057910) should be considered in Caucasian subjects. CYP2C19 (especially $* 2$ and $* 17$ ) and ABCB1 polymorphisms may also be considered for the evaluation of the patient. Additionally, serum phenytoin levels would be helpful to understand the contribution of genetic polymorphisms on the pharmacokinetics of phenytoin in the patients presenting side effects like DRESS syndrome.

The author declares no conflict of interest.

\section{References}

1. Anil H, Harmanci K, Tekin RT, Kocak A (2017): Presence of a single nucleotide polymorphism (RS3758581) in a boy with DRESS syndrome. Cent Eur J Immunol 42: 409-411.

2. Erdem SB, Nacaroglu HT, Bag O, et al. (2015): DRESS syndrome associated with type 2 diabetes in a child. Cent Eur J Immunol 40: 493-496.

3. Sandberg M, Yasar U, Stromberg P, et al. (2002): Oxidation of celecoxib by polymorphic cytochrome P450 2C9 and alcohol dehydrogenase. Br J Clin Pharmacol 54: 423-429.

4. Babaoglu M, Yasar U, Sandberg M, et al. (2004): CYP2C9 genetic variants and losartan oxidation in a Turkish population. Eur J Clin Pharmacol 60: 337-342.

5. Yampayon K, Sukasem C, Limwongse C, et al. (2017): Influence of genetic and non-genetic factors on phenytoin-induced severe cutaneous adverse drug reactions. Eur J Clin Pharmacol 73: 855-865.

6. Gumus E, Karaca O, Babaoglu MO, et al. (2012): Evaluation of lansoprazole as a probe for assessing cytochrome P450 2C19 activity and genotype-phenotype correlation in childhood. Eur J Clin Pharmacol 68: 629-636.

7. Brandolese R, Scordo MG, Spina E, et al. (2001): Severe phenytoin intoxication in a subject homozygous for CYP2C9*3. Clin Pharmacol Ther 70: 391-394.

8. Shaheen U, Prasad DK, Sharma V, et al. (2014): Significance of MDR1 gene polymorphism C3435T in predicting drug response in epilepsy. Epilepsy Res 108: 251-256. 